wirken. Trotzdem ist die Druckdifferenz in der Umgebung des Nullpunktes noch sehr bedeutend. Mit kleiner werdendem $\tau$ werden die Druckkurven immer höher (vgl. die Maßstäbe in den Abbildungen!). Es bilden sich, wenn $\tau \ll a_{/} / c$ ist, für $t>\tau$ auch scharfe Extrema aus. Interessant ist ferner die Beziehung zwischen der Stoßdauer $\tau$ und dem Verhältnis $a / c$. Ist $\tau=4 \mathrm{na} / \mathrm{c}$, d. h. ist der Stoß gerade dann beendet, wenn die von $r=a, \vartheta=0$ ausgehende Anfangsstörung nach $n$-maliger Reflexion am Gegenpol wieder zum Ausgangspunkt zurückgekehrt ist, so wird die für $t>\tau$ nach der Mitte vorstoßende Druckfront besonders stark (vgl. Abb. 9, 10). Ist dagegen $\tau=(4 n+2) a / c$, d. h. ist eine von $r=a, \vartheta=0$ ausgehende Welle gerade bei $r=a, \vartheta=\pi$ angekommen, so schwächt sich die nach der Mitte vorstoßende Welle schnell ab (vgl. Abb. 8). Abb. 7 zeigt einen
Druckverlauf, wenn $\tau=a / c$ ist. Bei einer Stoßkraft der Form einer Gaußschen Fehlerkurve:

$$
\begin{gathered}
\dot{v}(t)=0 \quad(t \leq 0) ; \quad \dot{v}(t)=2 v_{0} / \tau \sin ^{2}(\pi t / \tau) \quad(0 \leq t \leq \tau) ; \\
\dot{v}(t)=0 \quad(t \geq \tau)
\end{gathered}
$$

bekommt man, wie eine numerische Auswertung zeigt, schärfere Extrema. Bis zum Zeitpunkt $t=\tau / 2$ ist keine vorstoßende Störung festzustellen; denn bis dahin baut sich nur in der Umgebung der Kugelschale ein Extremum auf - entsprechend der stetigen Stoßkraft —, während der Druck in Richtung zum Kugelmittelpunkt rasch abklingt (Stauungsvorgang). Erst von $t=\tau$ ab stoßen Wellen ins Innere vor, aber dann mit stark ausgeprägten Extrema.

Hrn. Prof. Dr.-Ing. H. T is chner bin ich für die Anregung zu dieser Arbeit sowie für zahlreiche fördernde Ratschläge und Diskussionen zu großem Dank verpflichtet.

\title{
NOTIZEN
}

\section{Über Assoziation von Dipolmolekülen in Flüssig- keiten und Lösungen aus Messungen der dielektrischen Relaxationszeit}

Von Erich Fis cher

Universität Tübingen und Forschungsstelle für Spektroskopie in der Max-Planck-Gesellschaft, Hechingen

(Z. Naturforschg. 5 a, 628-629 [1950]; eingeg. am 11. Nov. 1950)

Die Erweiterung der Onsagerschen Theorie des inneren Feldes auf die Relaxationserscheinung der Dispersion und Absorption von Kurzwellen in Dipolfüssigkeiten ${ }^{1}$ ermöglicht Aussagen über die Assoziation polarer Moleküle. Dabei ist wesentlich, daß die Relaxationszeiten von Dipolmolekülen für den assoziationsfreien Zustand sehr verdünnter Auflösung in unpolaren Flüssigkeiten durch systematische Untersuchung bekannt sind, und zwar in ihrer Abhängigkeit von Molekül-Volumen, -Form und innermolekularer Beweglichkeit ${ }^{2}$. Auch an den meisten der zu H-Brücken-Assoziation befähigten Moleküle läßt sich durch Messungen in verdünnter Lösung die Relaxationszeit $\tau$ des entassoziierten Moleküls bestimmen und durch Vergleich mit den bekannten Relaxationszeiten anderer Moleküle etwas über die innermolekulare Beweglichkeit des zu Assoziation fähigen Dipols aussagen. Dieser $\tau$-Wert für das verdünnt gelöste Molekül ist dann die Ausgangsbasis, welche ermöglicht, bei höheren Konzentrationen bis zur reinen Flüssigkeit den Beginn und das Fortschreiten der Assoziation festzustellen aus der damit verbundenen Zunahme der Relaxationszeit, die gerade als

1 E. F is c h e r, Ann. Physik 6, 117 [1949].

2 s. E. F is ch e r, Z. Naturforschg. 4 a, 707 [1949] und die dort unter 3, 4, 5 zitierten Arbeiten.

3 E. F is c h e r, Physiker-Tagung in Bonn, Sept. 1949 Physiker-Tagung Württembg.-Baden, Freiburg, Dez. 1949.
Folge der durch die Assoziation bewirkten Einschränkung der innermolekularen Beweglichkeit und der Volumenvergrößerung der zu orientierenden Partikeln auftritt. Einige Ergebnisse solcher Untersuchungen, von denen z. Tl. bereits in Vorträgen ${ }^{3}$ berichtet wurde, seien kurz mitgeteilt.

Als unpolares Lösungsmittel diente $\mathrm{CCl}_{4}$, in welchem $\tau$-Komplexe zwischen Lösungsmittel und Gelöstem sich nicht bemerkbar machen ${ }^{4}$. Gemessen wurde aus dem dielektrischen Verlust im Meterwellengebiet die „effektive Relaxationszeit" $5(\tau)$, die sich, falls mehrere Relaxationszeiten vorhanden, in bestimmter Weise aus den Einzelwerten zusammensetzt.

Bei Chlorbenzol und $\alpha$-Bromnaphthalin als Beispiele nicht assoziierender Moleküle bleibt bei allen Konzentrationen (in $\mathrm{CCl}_{4}$ ) bis zur reinen Flüssigkeit $\tau / \eta$ weitgehend konstant $\left(\tau \sim \frac{\eta V}{k T} f ; V=\right.$ Partikelvolumen, $\eta=$ Viskosität, $f=$ Funktion der Form und inneren Beweglichkeit des Moleküls), wenn $\tau$ unter Berücksichtigung des Ons a g e r Feldes ausgewertet wird.

Phenol beginnt bei Konzentrationen unter 0,02 (Molenbruch) mit einer Relaxationszeit $\left(0,9 \cdot 10^{-11} \mathrm{sec}\right)$, die (im Vergleich zu Chlorbenzol $\left.1,2 \cdot 10^{-11} \mathrm{sec}\right)$ eine gewisse, wenn auch nicht völlig freie Beweglichkeit des OH-Dipols gegen den Phenylrest erkennen läßt. Es folgt dann sofort (besonders stark zwischen 0,05 und 0,1) ein starker Anstieg als Folge der Assoziation. Aus dem Verlauf von $\tau / \eta$ und seiner Temperaturabhängigkeit folgen mittiere Zähligkeiten und eine Assoziationsenergie, die im wesentichen den UR-spektroskopisch 6 im Gebiet bis 0,25 ermittelten

4 W. L ü t t k e u. R. M e cke, Z. Elektrochem. angew. physik. Chem. 53, 244 [1949].

5 E. F i s c h e r, Physik. Z. 40, 645 [1939], S. 648 und 2.

${ }_{6}$ R. M e c k e, Z. Elektrochem. angew. physik. Chem. 52, 269 [1948]. 
entsprechen. Bei höheren Konzentrationen, bei denen die UR-Methode nicht weiterführt, überschreitet $\tau / \eta$ ein flaches Maximum. Das zeigt, daß dann wieder kleinere Relaxationszeiten wirksam werden, dadurch, daß eine Eigenbeweglichkeit der Einzelglieder der Assoziationsketten stattfindet oder die Lebensdauer der Komplexe kleiner wird. Die Dispersionskurve dürfte sich deshalb von einer stark verbreiterten Form, welche sie im Falle verschieden großer Einzelrelaxationszeiten der verschiedenen Komplexe annimmt, wieder der Form annähern, die für eine einzige Relaxationszeit kennzeichnend ist.

Bei den $o$-substituierten Phenolen, bei welchen die Möglichkeit zu innermolekularer $\mathrm{H}$-Brücke gegeben ist und an welchen $\mathrm{Mecke}$ und $\mathrm{Reute} \mathrm{r}^{7}$ die statische Polarisation (Dipolmoment) untersuchten, läßt die Relaxationsuntersuchung in Ergänzung der Dipolmomentmessungen besonders feine Unterschiede erkennen. $o$-Kresol zeigt das Verhalten von Phenol im abgeschwächten Maß. Bei $o$-Chlorphenol, Salicylaldehyd und $o$-Nitrophenol steigt $\tau / \eta$ auf das 1,5- bis 2-fache des Anfangswertes an und bleibt dann im wesentlichen konstant. Es kommt also zu einer schwachen, durch die Möglichkeit zur Bildung innerer H-Brücken eingeschränkten Assoziation. Die Anfangswerte ( $\infty$-Verdünnung) weisen, besonders bei $o$ Chlorphenol und Salicylaldehyd, darauf hin, daß trotz des $o$-Substituenten innermolekulare Dipolbeweglichkeit besteht. Salicylsäuremethylester beginnt bei kleinen Konzentrationen mit einem $\tau$-Wert, der einem starr mit dem Phenylrest verbundenen Dipol als Folge der inneren $\mathrm{H}$-Brücke der beiden Substituenten $\left(\cdot \mathrm{OH}\right.$ und $-\mathrm{COOCH}_{3}$ ) entspricht. Es gleicht in seinem Verhalten $-\tau / \eta$ bleibt weitgehend konstant - Chlorbenzol und $\alpha$-Bromnaphthalin, die nicht assoziieren.

An Nitrobenzol in $\mathrm{CCl}_{4}$ läßt die Relaxationsuntersuchung erkennen, daß es, besonders bei mittleren Konzentrationen (Maximum von $\tau / \eta$ ), assoziiert, wenn auch schwächer als Moleküle mit $\mathrm{OH}-$ Dipolen (Phenol). Auch bei reinem Nitrobenzol ist $\tau / \eta$ größer als in verdünnter Lösung, woraus auf Komplexe längerer Lebensdauer zu schließen ist. Löst man Nitrobenzol verdünnt in Benzol, so findet bereits eine Erhöhung der Relaxationszeit durch Komplexbildung mit Benzol statt; der Vergleich der Relaxationszeit von reinem Nitrobenzol mit diesem Wert konnte daher ein normales Verhalten von Nitrobenzol vortäuschen ${ }^{8}$. Die Assoziation von reinem Nitrobenzol ist ein Grund für die verschiedentlich gefundene Verbreiterung des Dispersionsgebietes; sie sollte nach unseren Ergebnissen bei einer Mischung mit $\mathrm{CCl}_{4}$ noch ausgeprägter werden. Benzonitril verhält sich dem Nitrobenzol ähnlich.

Für ein Molekül, das sich in unpolarem Lösungsmittel (Benzol) nur sehr wenig löst $(0,003)$ und in diesem Bereich bereits starke Assoziation zeigt, erwies sich Imidazol als ein demonstratives Beispiel. $\tau$ steigt (bei $25^{\circ} \mathrm{C}$ ) bis zur Konzentration 0,003 auf etwa das 40-fache des Wertes für unendliche Verdünnung, und die Temperaturabhängigkeit läßt auf eine besonders hohe Assoziationsenergie schließen. Schließlich sei $p$-Aminoacetophenon erwähnt, bei welchem

7 R. Me cke u. A. Re uter, Z. Naturforschg. 4a, 368 [1949].

8 E. F i s ch e r, Z. Physik 127, 49 [1949]. bis zur Grenze seiner Löslichkeit in Benzol $(\sim 0,01)$ ein kcnstantes Dipolmoment gemessen wird, weshalb man bisher sich berechtigt glaubte, auf das Fehlen einer Assoziation zu schließen. Die empfindlichere Relaxationsuntersuchung ergibt trotzdem, daß $\tau$ etwa auf das Doppelte des dem Einermolekül zukommenden Wertes ansteigt, also Assoziation (ohne wesentliche Momentänderung) vorliegt.

In vielen Fällen wird die Messung der Relaxationszeit wesentlich zum Studium von Assoziationserscheinungen beitragen können. Im einzelnen wird die Mitteilung des experimentellen Teils und die quantitative Diskussion in ausführlichen Veröffentlichungen erfolgen.

\section{Gewinnung eines praktisch trägerfreien Radio-Kupfer- Präparates ${ }^{64} \mathrm{Cu}$ von hoher Aktivität aus $\mathrm{Cu}-\mathrm{Phthalocyanin}$}

Von W. He r r und H. Göt te

Max-Planck-Institut für Chemie, Mainz

(Z. Naturforschg. 5 a, 629-630 [1950]; eingeg. am 7. Nov. 1950)

Von den beiden zur Gewinnung von ${ }^{64} \mathrm{Cu}$-Präparaten anwendbaren Kernprozessen ${ }_{30}^{64} \mathrm{Zn}(\mathrm{n}, \mathrm{p}){ }_{29}^{64} \mathrm{Cu}^{1}$ und ${ }_{29}^{63} \mathrm{Cu}(\mathrm{n}, \gamma){ }_{29}^{64} \mathrm{Cu}$ gibt der zweite wegen seines größeren Wirkungsquerschnittes die größeren Aktivitäten. Versuche, das so entstandene ${ }^{64} \mathrm{Cu}$ von nicht aktiviertem nach dem Verfahren von $\mathrm{S} z$ i l a rd und $\mathrm{C}$ h a l m e r s 2 abzutrennen, führten bei Bestrahlung der $\mathrm{Cu}$-salizylaldehyd$o$-phenylendiimin-Komplexverbindung zum Erfolg ${ }^{3}$. Der Verwendung der komplizierten und teuren Verbindung stehen aber erhebliche Schwierigkeiten entgegen.

Es war nun zu erwarten, daß die stabilen Komplexverbindungen der Phthalocyanine geeignete Substanzen für ein derartiges Abtrennverfahren sein könnten. Diese Stoffklasse zeichnet sich durch große Beständigkeit gegen chemische Agenzien und hohe Temperatur aus ${ }^{4}$. Es ist durch Röntgenuntersuchungen 5 bekannt, daß die Atome ihrer Moleküle alle in einer Ebene angeordnet sind und daß das komplexbildende Metallatom sich in der Mitte des Moleküles befindet (vgl. Formel Abb. 1). Aus diesem Grunde war anzunehmen, daß das beim Neutroneneinfang durch $\gamma$-Rückstoß angestoßene Zentralatom nicht durch andere Atome oder Atomgruppen am Austritt aus dem Molekül gehindert 6 wird.

Diese Hypothese bestätigte sich beim $\mathrm{Cu}-\mathrm{Phthc}$ aufs beste. Die Ausbeuten an Radiokupfer betragen bis zu $90 \%$.

1 O. Erbacher, W. Herr u. U. Egidi, Z. anorg. allg. Chem. 256, 41 [1948]. - H. J. Born u. U. Drehm a n n, Naturwiss. 32, 159 [1944].

2 L.Szilard u. T. A. Chalmers, Nature [London] 134, 462 [1934].

3 R. B. Duffield u. M. Calvin, J. Amer. chem. Soc. 68, 1129 [1946].

4 R. P. Linstead, C. T. Byrne, A. R. Lowe u. a., J. chem. Soc. [London] 1934, 1016.

5 J. M. R oberts on u. J. Wo od wood, J. chem. Soc. [London] 1937, 219.

6 P. Sue u. G. Kayas, J. Chim. Physique 45, 188 [1948]. 